Discrete Comput Geom 27:3-28 (2002)

DOI: $10.1007 / \mathrm{s} 00454-001-0049-4$

\title{
Infinitesimal Hopf Algebras and the cd-Index of Polytopes*
}

\author{
M. Aguiar \\ Centre de Recherches Mathématiques, Université de Montréal, \\ C.P. 6128, Succ. Centre-ville, Montréal, Québec, Canada H3C 3J7 \\ maguiar@math.tamu.edu
}

\begin{abstract}
Infinitesimal bialgebras were introduced by Joni and Rota [JR]. The basic theory of these objects was developed in [A1] and [A2]. In this paper we present a simple proof of the existence of the cd-index of polytopes, based on the theory of infinitesimal Hopf algebras.

For the purpose of this work, the main examples of infinitesimal Hopf algebras are provided by the algebra $\mathcal{P}$ of all posets and the algebra $k\langle\mathbf{a}, \mathbf{b}\rangle$ of noncommutative polynomials. We show that $k\langle\mathbf{a}, \mathbf{b}\rangle$ satisfies the following universal property: given a graded infinitesimal bialgebra $A$ and a morphism of algebras $\zeta_{A}: A \rightarrow k$, there exists a unique morphism of graded infinitesimal bialgebras $\psi: A \rightarrow k\langle\mathbf{a}, \mathbf{b}\rangle$ such that $\zeta_{1,0} \psi=\zeta_{A}$, where $\zeta_{1,0}$ is evaluation at $(1,0)$. When the universal property is applied to the algebra of posets and the usual zeta function $\zeta_{\mathcal{P}}(P)=1$, one obtains the $\mathbf{a b}$-index of posets $\psi: \mathcal{P} \rightarrow k\langle\mathbf{a}, \mathbf{b}\rangle$.

The notion of antipode is used to define an analog of the Möbius function of posets for more general infinitesimal Hopf algebras than $\mathcal{P}$, and this in turn is used to define a canonical infinitesimal Hopf subalgebra, called the eulerian subalgebra. All eulerian posets belong to the eulerian subalgebra of $\mathcal{P}$. The eulerian subalgebra of $k\langle\mathbf{a}, \mathbf{b}\rangle$ is precisely the algebra spanned by $\mathbf{c}=\mathbf{a}+\mathbf{b}$ and $\mathbf{d}=\mathbf{a b}+\mathbf{b a}$. The existence of the $\mathbf{c d}$-index of eulerian posets is then an immediate consequence of the simple fact that eulerian subalgebras are preserved under morphisms of infinitesimal Hopf algebras.

The theory also provides a version of the generalized Dehn-Sommerville equations for more general infinitesimal Hopf algebras than $k\langle\mathbf{a}, \mathbf{b}\rangle$.
\end{abstract}

\section{Introduction}

Let $P$ be a convex polytope of affine dimension $\operatorname{dim} P=n+1$. For each subset $\mathrm{S}$ of $\{1, \ldots, n\}$, let $f_{\mathrm{S}}(P)$ denote the number of chains of faces $F_{1} \subset \cdots \subset F_{i}$ of $P$ such

* This research was supported by a Postdoctoral Fellowship at the Centre de Recherches Mathématiques and Institut des Sciences Mathématiques, Montréal, Québec. 
that $\left\{\operatorname{dim} F_{1}, \ldots, \operatorname{dim} F_{i}\right\}=\mathrm{S}$. The flag vector of $P$ is the $2^{n}$-dimensional vector with coordinates $f_{\mathrm{S}}(P)$ indexed by the subsets of $\{1, \ldots, n\}$.

An important result of Bayer and Billera describes the linear relations that hold among the flag vectors of all convex polytopes [BB, Theorem 2.1]. These are known as the generalized Dehn-Sommerville equations. There is one such relation associated to every subset $\mathrm{S}$ of $\{1, \ldots, n\}$ and a maximal interval $[a, b]$ of $\{1, \ldots, n\} \backslash \mathrm{S}$, as follows:

$$
(-1)^{a-1} f_{\mathrm{S}}+\sum_{i=a}^{b}(-1)^{i} f_{\mathrm{S} \cup\{i\}}+(-1)^{b+1} f_{\mathrm{S}}=0 .
$$

These generalize Euler's relation among the number of faces of a convex polytope, which is the relation that corresponds to $\mathrm{S}=\emptyset$ (and $a=1, b=n$ ).

The poset of faces of a convex polytope is an example of an eulerian poset (see Section 6). Bayer and Billera showed that these relations hold among the flag vectors of all eulerian posets.

Later, Bayer, Klapper and Fine noted that one could encode the existence of these relations by means of what appears to be a simple change of notation. First, one rewrites the flag vector as a homogeneous polynomial of two noncommuting variables $\mathbf{a}$ and $\mathbf{b}$, whose coefficients are the entries of the vector. This defines, for every graded poset $P$, a polynomial in $\mathbf{a}$ and $\mathbf{b}$ called its $\mathbf{a b}$-index . It turns out that the flag vector of $P$ satisfies the generalized Dehn-Sommerville equations if and only if its $\mathbf{a b}$-index can be expressed as polynomial in the variables $\mathbf{c}=\mathbf{a}+\mathbf{b}$ and $\mathbf{d}=\mathbf{a b}+\mathbf{b a}[\mathrm{BK}$, Theorem 4]. This new polynomial is called the cd-index . By the result of Bayer and Billera, it is defined for all eulerian posets.

More recent work of Ehrenborg and Readdy suggested that there might be more than meets the eye to encoding the flag vector as a polynomial. They showed that one can define two natural infinitesimal bialgebra structures (see Section 2), one consisting of posets, the other of polynomials; one may view the ab-index as a map $\psi$ from the former to the latter, and it turns out that these structures are preserved under $\psi$ [ER, Proposition 3.1].

In this work we make full use of this algebraic structure in order to produce a new proof of the existence of the cd-index of eulerian posets. The crucial algebraic notion that must be considered to obtain the existence of the cd-index is the notion of antipodes for infinitesimal bialgebras. This was defined and studied in [A1]. It allows us to define analogs of the Möbius function and eulerian posets for other infinitesimal bialgebras than that of posets. The analog of eulerian posets for the infinitesimal bialgebra of polynomials is precisely the polynomials on $\mathbf{c}$ and $\mathbf{d}$. The existence of the cd-index is then obtained from the simple fact that eulerian subalgebras are preserved under morphisms of infinitesimal bialgebras, applied to the ab-index . This is done in Section 6.

Each eulerian subalgebra is defined by a canonical set of equations, which may be regarded as a version of the generalized Dehn-Sommerville equations for arbitrary infinitesimal Hopf algebras. For the algebra of polynomials, these equations are not exactly the same as the equations of Bayer and Billera, although they have the same solutions. We present these equations in Section 7.

In Sections 4 and 5 we find that the ab-index can be defined by means of a very simple universal property satisfied by the infinitesimal Hopf algebra of polynomials. We 
also show that other variants of the ab-index can be obtained similarly from the same universal property. Thus, we find that the notion of infinitesimal Hopf algebra provides a solid conceptual base for the theory of the ab-index .

Alternative approaches to flag vectors, the ab-index and $\mathbf{c d}$-index can be found in the recent works [BL], [BMSV] and [E3].

I would like to express my gratitude to Richard Ehrenborg for introducing me to his work on these topics.

Notation. $k$ denotes a fixed field, often omitted from the notation. Sum symbols are omitted from Sweedler's notation: we write $\Delta(a)=a_{1} \otimes a_{2}$ when $\Delta$ is a coassociative comultiplication.

\section{Infinitesimal Hopf Algebras. Examples}

Definition 2.1. An infinitesimal bialgebra (abbreviated $\varepsilon$-bialgebra) is a triple $(A$, $m, \Delta)$ where

$(A, m)$ is an associative algebra (possibly without unit),

$(A, \Delta)$ is a coassociative coalgebra (possibly without counit)

and, for each $a, b \in A$,

$$
\Delta(a b)=a b_{1} \otimes b_{2}+a_{1} \otimes a_{2} b
$$

In other words, the comultiplication $\Delta: A \rightarrow A \otimes A$ is required to be a derivation of the algebra $A$ with values on the $A$-bimodule $A \otimes A$, or, equivalently, the multiplication $m: A \otimes A \rightarrow A$ is required to be a coderivation of the coalgebra $A$.

Infinitesimal bialgebras were introduced by Joni and Rota [JR, Section XII]. Ehrenborg and Readdy have called them newtonian coalgebras [ER]. Infinitesimal Hopf algebras were introduced in [A1].

Definition 2.2. An infinitesimal bialgebra $A$ is called an infinitesimal Hopf algebra (abbreviated $\varepsilon$-Hopf algebra) if there is a map $S: A \rightarrow A$ with the property that

$$
S\left(a_{1}\right) a_{2}+S(a)+a=0=a_{1} S\left(a_{2}\right)+a+S(a), \quad \forall a \in A .
$$

In this case, the map $S$ is unique. It is called the antipode of $A$. Among other properties, it satisfies

$$
\begin{aligned}
S(1) & =-1 \quad \text { (if there is a unit element), } \\
S(a b) & =-S(a) S(b), \\
S\left(a_{1}\right) \otimes S\left(a_{2}\right) & =-S(a)_{1} \otimes S(a)_{2} .
\end{aligned}
$$

These assertions are proven in Section 3 of [A1]. 
Example 2.3. (1) Let $Q$ be an arbitrary quiver (oriented graph). Then the path algebra $k Q$ carries a canonical $\varepsilon$-bialgebra structure. Recall that $k Q=\bigoplus_{n=0}^{\infty} k Q_{n}$ where $Q_{n}$ is the set of paths $\gamma$ in $Q$ of length $n$ :

$$
\gamma: e_{0} \stackrel{a_{1}}{\rightarrow} e_{1} \stackrel{a_{2}}{\rightarrow} e_{2} \stackrel{a_{3}}{\rightarrow} \cdots e_{n-1} \stackrel{a_{n}}{\rightarrow} e_{n} .
$$

In particular, $Q_{0}$ is the set of vertices and $Q_{1}$ is the set of arrows. The multiplication is concatenation of paths whenever possible; otherwise is zero. The comultiplication is defined on a path $\gamma=a_{1} a_{2} \cdots a_{n}$ as above by

$$
\Delta(\gamma)=e_{0} \otimes a_{2} a_{3} \cdots a_{n}+a_{1} \otimes a_{3} \cdots a_{n}+\cdots+a_{1} \cdots a_{n-1} \otimes e_{n} .
$$

In particular, $\Delta(e)=0$ for every vertex $e \in Q_{0}$ and $\Delta(a)=s(a) \otimes t(a)$ for every arrow $a \in Q_{1}$.

The path algebra $k Q$ is an $\varepsilon$-Hopf algebra. The antipode is uniquely determined, in view of 2.4 , by

$$
\begin{aligned}
& S(e)=-e, \quad \forall e \in Q_{0}, \quad \text { and } \\
& S(a)= \begin{cases}e-a & \text { if } \quad s(a)=t(a)=e, \quad \forall a \in Q_{1} . \\
-a & \text { if } \quad s(a) \neq t(a),\end{cases}
\end{aligned}
$$

The path $\varepsilon$-Hopf algebra corresponding to the quiver

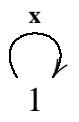

is the polynomial algebra $k[\mathbf{x}]$. Under the standard identification $k[\mathbf{x}] \otimes k[\mathbf{x}] \cong k[\mathbf{x}, \mathbf{y}]$, the comultiplication is the divided difference operator

$$
\Delta(f(\mathbf{x}))=\frac{f(\mathbf{x})-f(\mathbf{y})}{\mathbf{x}-\mathbf{y}} .
$$

This was the original example of $\varepsilon$-bialgebra in [JR]. The antipode is

$$
S(f(\mathbf{x}))=-f(\mathbf{x}-1) .
$$

Of particular relevance to our study of the cd-index is the $\varepsilon$-Hopf algebra of noncommutative polynomials $k\langle\mathbf{a}, \mathbf{b}\rangle$. This is the $\varepsilon$-Hopf algebra corresponding to the quiver

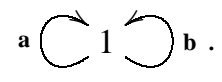

The comultiplication is the unique derivation such that

$$
\Delta(\mathbf{a})=\Delta(\mathbf{b})=1 \otimes 1 .
$$

The antipode is given on any noncommutative polynomial $p(\mathbf{a}, \mathbf{b})$ by

$$
S(p(\mathbf{a}, \mathbf{b}))=-p(\mathbf{a}-1, \mathbf{b}-1) .
$$


(2) The algebra $\mathcal{P}$ of posets is defined as follows. As a vector space, $\mathcal{P}$ has a basis consisting of the isomorphism classes of all finite graded posets $P$. We say that a finite poset $P$ is graded if it possesses a top element $1_{P}$ and a bottom element $0_{P}$, with $0_{P} \neq 1_{P}$, and all maximal chains in $P$ have the same length. ${ }^{1}$ This common length is called the rank of the poset.

The product of two such posets $P$ and $Q$ is the poset

$$
P Q=\left(P-\left\{1_{P}\right\}\right) \cup\left(Q-\left\{0_{Q}\right\}\right),
$$

(disjoint union), where

$$
x \leq y \quad \text { iff }\left\{\begin{array}{l}
x, y \in P \text { and } x \leq y \text { in } P, \\
x, y \in Q \text { and } x \leq y \text { in } Q, \quad \text { or } \\
x \in P \text { and } y \in Q .
\end{array}\right.
$$

This algebra possesses a unit element, namely the poset $B_{1}=\{0<1\}$. Moreover, $\mathcal{P}$ is an $\varepsilon$-bialgebra with comultiplication

$$
\Delta(P)=\sum_{0_{P}<x<1_{P}}\left[0_{P}, x\right] \otimes\left[x, 1_{P}\right] .
$$

Here, and in everything that follows, if $x$ and $y$ are two elements of a poset $P$, then $[x, y]$ denotes the poset $\{z \in P / x \leq z \leq y\}$.

This $\varepsilon$-bialgebra was first considered by Ehrenborg and Hetyei $[\mathrm{EH}]$, and further studied by Billera, Ehrenborg and Readdy in connection with the cd-index of polytopes [ER], [BE]. This study is deepened in this work. In Section 3 we show that $\mathcal{P}$ is an $\varepsilon$-Hopf algebra with antipode

$$
S(P)=\sum_{c}(-1)^{\ell(c)} P_{c}
$$

where the sum is over all chains $c=\left\{0_{P}=x_{0}<x_{1}<\cdots<x_{k-1}<x_{k}=1_{P}\right\}$, $\ell(c)=k$ is the length of the chain and $P_{c}$ is the following subposet of $P$ :

$$
P_{c}=\left\{x \in P / x_{i-1}<x<x_{i} \text { for some } i=1, \ldots, k\right\} \cup\left\{0_{P}, 1_{P}\right\} .
$$

We also relate the antipode of $\mathcal{P}$ to the Möbius function of posets. In Section 6 we obtain the existence of the cd-index from further exploitation of the $\varepsilon$-Hopf algebra structure of $\mathcal{P}$.

Remark. There is a way of constructing an ordinary Hopf algebra $H$ from isomorphism classes of posets, similar to the construction of the $\varepsilon$-bialgebra $\mathcal{P}$ above, that appears in several places in the literature [AF], [E1], [S1]; see also [BS]. As a coalgebra, $H$ is obtained from $\mathcal{P}$ by formally adjoining a counit; however, the algebra structures of $H$ and $\mathcal{P}$ are of a fairly different nature: while the product of $H$ is the cartesian product of

\footnotetext{
${ }^{1}$ The assumption that the posets be graded is not necessary for obtaining an $\varepsilon$-Hopf algebra, but it is required for the definition of the $\mathbf{a b}$-index .
} 
posets, that of $\mathcal{P}$ is (essentially) the ordinal sum. The $\varepsilon$-bialgebra structure of $\mathcal{P}$ is suitable for the algebraic construction of the ab-index and cd-index of posets that we propose in this work. An approach to the ab-index and cd-index via ordinary Hopf algebras is presented in [BMSV].

(3) The algebra $\mathcal{S}$ of sets is defined as follows. As a vector space, $\mathcal{S}=\bigoplus_{n=0}^{\infty} \mathcal{S}_{n}$, where the vector space $\mathcal{S}_{n}$ has a basis consisting of all subsets of $\{1,2, \ldots, n\}$. The product of two sets $S \in \mathcal{S}_{n}$ and $\mathrm{T} \in \mathcal{S}_{m}$ is the set

$$
\mathrm{ST}=\mathrm{S} \cup(n+\mathrm{T}) \in \mathcal{S}_{n+m} .
$$

In other words, the elements of T are all shifted by $n$ in order to make the resulting union disjoint.

In order to describe the comultiplication, we introduce the following notation. For $\mathrm{S} \subseteq\{1,2, \ldots, n\}$ and $0 \leq i<j \leq n+1$, let

$$
\mathrm{S}(i, j)=\mathrm{S} \cap\{i+1, i+2, \ldots, j-1\}-i \subseteq\{1,2, \ldots, j-i-1\} .
$$

In other words, $\mathbf{S}(i, j)$ consists of those elements of $S$ that lie strictly between $i$ and $j$, but shifted by $-i$ in order to make the resulting intersection a subset of $\{1,2, \ldots, j-i-1\}$. The comultiplication on a basis element $S \in \mathcal{S}_{n}$ is

$$
\Delta(\mathrm{S})=\sum_{i \in \mathrm{S}} \mathrm{S}(0, i) \otimes \mathrm{S}(i, n+1) \in \sum_{i=1}^{n} \mathcal{S}_{i-1} \otimes \mathcal{S}_{n-i}
$$

In order to distinguish between elements of different $\mathcal{S}_{n}$ 's we sometimes use $S_{n}$ to denote the element of $\mathcal{S}_{n}$ corresponding to a subset $S \subseteq\{1,2, \ldots, n\}$. For instance, for each $n$ there is a different element $\emptyset_{n} \in \mathcal{S}_{n}$, corresponding to the empty subset of $\{1,2, \ldots, n\}$. Some values of the comultiplication are

$$
\Delta\left(\emptyset_{n}\right)=0, \quad \forall n \geq 0, \quad \text { and } \quad \Delta\left(\{2,3\}_{3}\right)=\emptyset_{1} \otimes\{1\}_{1}+\{2\}_{2} \otimes \emptyset_{0} .
$$

$\mathcal{S}$ is an $\varepsilon$-Hopf algebra. The antipode is explicitly given on $\mathrm{S} \in \mathcal{S}_{n}$ by

$$
S(\mathrm{~S})=-\sum_{\mathrm{T} \subseteq \mathrm{S}}(-1)^{\# \mathrm{~T}} \mathrm{~S} \backslash \backslash \mathrm{T},
$$

where $S \backslash \backslash T$ is a shifted version of the usual set difference $S \backslash T$. In order to describe this construction, define $f: \mathbb{N} \rightarrow \mathbb{N}$ by $f(i)=\#(\{1,2, \ldots, i\} \cap \mathrm{T})$. Then

$$
\mathrm{S} \backslash \backslash \mathrm{T}=\{i-f(i) / i \in \mathrm{S} \backslash \mathrm{T}\} \in \mathcal{S}_{n-\# \mathrm{~T}} .
$$

For instance, if $S=\{1,3,4,7,8,10\}$ and $T=\{3,4,8\}$, then $S \backslash \backslash T=\{1-0,7-2,10-$ $3\}=\{1,5,7\}$.

The $\varepsilon$-Hopf algebra $\mathcal{S}$ is studied in detail in Section 4 .

(4) The free algebra $A=k\left\langle\mathbf{x}_{1}, \mathbf{x}_{2}, \mathbf{x}_{3}, \ldots\right\rangle$ is an $\varepsilon$-Hopf algebra with comultiplication

$$
\Delta\left(\mathbf{x}_{n}\right)=\sum_{i=0}^{n-1} \mathbf{x}_{i} \otimes \mathbf{x}_{n-1-i}=1 \otimes \mathbf{x}_{n-1}+\mathbf{x}_{1} \otimes \mathbf{x}_{n-2}+\cdots+\mathbf{x}_{n-1} \otimes 1
$$


and antipode

$$
S\left(\mathbf{x}_{n}\right)=\sum_{k=1}^{n+1}(-1)^{k} \sum_{\substack{n_{1}+\cdots+n_{k}=n+1 \\ n_{i}>0}} \mathbf{x}_{n_{1}-1} \mathbf{x}_{n_{2}-1} \cdots \mathbf{x}_{n_{k}-1}
$$

(we set $\mathbf{x}_{0}=1$ ). $\Delta$ and $S$ are then uniquely determined by (2.1) and (2.4), and the axioms are satisfied.

More examples of $\varepsilon$-bialgebras appear in [A1] and [A2]. We close this section by stating some general properties of $\varepsilon$-bialgebras. Proofs and more results can be found in [A1].

If an $\varepsilon$-bialgebra has a unit $1 \in A$, then $\Delta(1)=0$. If an $\varepsilon$-bialgebra has both a unit $1 \in A$ and a counit $\varepsilon \in A^{*}$, then $A=0$.

If $C$ is a coalgebra and $A$ is an algebra, then the set $\operatorname{Hom}_{\mathrm{k}}(C, A)$ is a monoid under the circular convolution product:

$$
f \circledast g=f * g+f+g \quad \text { or } \quad(f \circledast g)(c)=f\left(c_{1}\right) g\left(c_{2}\right)+f(c)+g(c) .
$$

The unit element of this monoid is the zero map. The antipode of an $\varepsilon$-Hopf algebra $A$ is, by definition, the inverse of the identity $i d: A \rightarrow A$ in $\operatorname{Hom}_{\mathrm{k}}(A, A)$.

A morphism of $\varepsilon$-bialgebras is a linear map $\psi: A \rightarrow B$ that is both a morphism of algebras and coalgebras:

$$
m_{B}(\psi \otimes \psi)=\psi m_{A} \quad \text { and } \quad(\psi \otimes \psi) \Delta_{A}=\Delta_{B} \psi .
$$

If $A$ and $B$ are $\varepsilon$-Hopf algebras and $\psi: A \rightarrow B$ a morphism of $\varepsilon$-bialgebras, then $\psi$ automatically preserves the antipodes: $\psi S_{A}=S_{B} \psi$.

Let $A$ be an $\varepsilon$-Hopf algebra and let $B$ be an algebra. If $f: A \rightarrow B$ is a morphism of algebras, then $f$ is invertible in $\operatorname{Hom}_{\mathrm{k}}(A, B)$ with respect to circular convolution, its inverse is $f S_{A}$. In addition, $-f S_{A}: A \rightarrow B$ is a morphism of algebras, by (2.4).

An $\varepsilon$-bialgebra $(A, m, \Delta)$ is called graded if there is a sequence $A_{n}$ of subspaces such that

$$
A=\bigoplus_{n=0}^{\infty} A_{n}, \quad m\left(A_{i} \otimes A_{j}\right) \subseteq A_{i+j} \quad \text { and } \quad \Delta\left(A_{n}\right) \subseteq \bigoplus_{i+j=n-1} A_{i} \otimes A_{j} .
$$

Note that it is required that $\Delta$ lowers degrees by 1 . In this case, $A$ is an $\varepsilon$-Hopf algebra with antipode

$$
S=\sum_{n=1}^{\infty}(-1)^{n} m^{(n-1)} \Delta^{(n-1)}, \quad \text { i.e. } \quad S(a)=\sum_{n=1}^{\infty}(-1)^{n} a_{1} a_{2} \cdots a_{n},
$$

where $\Delta^{(n-1)}(a)=a_{1} \otimes a_{2} \otimes \cdots \otimes a_{n}$ denotes the comultiplication iterated $n-1$ times. This result guarantees the existence of the antipode for the $\varepsilon$-bialgebras of Examples 2.3(1)-(4), since they are graded.

\section{The $\varepsilon$-Hopf Algebra of Posets}

In this section we use the general properties of $\varepsilon$-bialgebras summarized above to relate the antipode of the $\varepsilon$-Hopf algebra $\mathcal{P}$ to the classical Möbius function of posets. 
First, note that $\mathcal{P}$ is graded in the sense of (2.7), by choosing for $\mathcal{P}_{n}$ the subspace spanned by all posets of rank $n+1$, i.e. those posets $P$ for which there is a maximal chain of the form $0_{P}<x_{1}<\cdots<x_{n}<1_{P}$.

Let $P \in \mathcal{P}$ be a poset. We have $\Delta(P)=\sum_{0_{P}<x<1_{P}}\left[0_{P}, x\right] \otimes\left[x, 1_{P}\right]$. Hence

$$
\Delta^{(n-1)}(P)=\sum_{0_{P}<x_{1}<\cdots<x_{n-1}<1_{P}}\left[0_{P}, x_{1}\right] \otimes\left[x_{1}, x_{2}\right] \otimes \cdots \otimes\left[x_{n-1}, 1_{P}\right] ;
$$

and, according to (2.8),

$$
S(P)=\sum_{n=1}^{\infty}(-1)^{n} \sum_{0_{P}<x_{1}<\cdots<x_{n-1}<1_{P}}\left[0_{P}, x_{1}\right]\left[x_{1}, x_{2}\right] \cdots\left[x_{n-1}, 1_{P}\right] .
$$

By rewriting this formula in terms of chains we obtain (2.6).

Consider the linear functionals $\zeta: \mathcal{P} \rightarrow k$ and $\mu: \mathcal{P} \rightarrow k$ defined by

$$
\zeta(P)=1, \quad \forall \text { poset } P \in \mathcal{P} \quad \text { and } \quad \mu=\zeta S .
$$

Note that $\zeta$ is a morphism of algebras. As recalled at the end of Section 2, it follows that $\zeta$ is circular invertible with inverse $\mu$, and that $-\mu$ is a morphism of algebras. Explicitly, the first fact says

$$
0=\mu \circledast \zeta=\mu * \zeta+\mu+\zeta .
$$

Evaluating at a poset $P \in \mathcal{P}$ we find

$$
\begin{aligned}
0=\sum_{0_{P}<x<1_{P}} \mu\left[0_{P}, x\right] \zeta\left[x, 1_{P}\right]+\mu(P)+\zeta(P) \\
\Rightarrow \mu\left[0_{P}, 1_{P}\right]=-1-\sum_{0_{P}<x<1_{P}} \mu\left[0_{P}, x\right] .
\end{aligned}
$$

This shows that $\mu$ is the usual Möbius function of posets (since this is its defining recursion).

Applying $\zeta$ to both sides of 3.1 we find

$$
\mu(P)=\sum_{n=1}^{\infty}(-1)^{n} \#\left\{0_{P}<x_{1}<\cdots<x_{n-1}<1_{P}\right\} ;
$$

the well-known formula of P. Hall giving the Möbius function in terms of numbers of chains.

The fact that $-\mu$ is a morphism of algebras gives us

$$
\mu(P Q)=-\mu(P) \mu(Q),
$$

another well-known property of the Möbius function and the product of posets under consideration.

As an illustration of the structure of $\mathcal{P}$, we close this section by explicitly describing a particular $\varepsilon$-Hopf subalgebra. The subspace of $\mathcal{P}$ spanned by all boolean posets $B_{n}$ (the poset of subsets of $\{1,2, \ldots, n\}$ ) is clearly a subcoalgebra of $\mathcal{P}$. It follows that the subalgebra $\mathcal{B}$ of $\mathcal{P}$ generated by all boolean posets is an $\varepsilon$-Hopf subalgebra. Moreover, it is easy to see that $\mathcal{B}$ is isomorphic to the $\varepsilon$-Hopf algebra of polynomials of Example 2.3(4), via $\left(\mathbf{x}_{n} \mapsto 1 /(n+1)\right.$ !) $B_{n+1}$. $\mathcal{P}$ itself is also a free algebra, but this is not important for us. 


\section{The Universal Property of the $\varepsilon$-Hopf Algebra of Sets}

The algebra of sets (Example 2.3(3)) comes equipped with a morphism of algebras $\zeta_{\mathcal{S}}: \mathcal{S} \rightarrow k$ defined on $\mathcal{S} \in \mathcal{S}_{n}$ by

$$
\zeta_{\mathcal{S}}(\mathrm{S})= \begin{cases}1 & \text { if } \quad \mathrm{S}=\emptyset_{n} \in \mathcal{S}_{n}, \\ 0 & \text { if } \quad \mathrm{S} \neq \emptyset_{n} .\end{cases}
$$

In this work we say that a coalgebra $(C, \Delta)$ is graded if $C=\bigoplus_{n=0}^{\infty} C_{n}$ with $\Delta\left(C_{n}\right) \subseteq$ $\sum_{i+j=n-1} C_{i} \otimes C_{j}$. This agrees with the terminology introduced before (2.7) for $\varepsilon$ bialgebras.

The pair $\left(\mathcal{S}, \zeta_{\mathcal{S}}\right)$ satisfies the following universal property, which reveals its central role in the theory.

Theorem 4.1. Let $C$ be a graded coalgebra and let $\zeta_{C}: C \rightarrow k$ be a linear functional. Then there exists a unique morphism of graded coalgebras $\psi: C \rightarrow \mathcal{S}$ such that

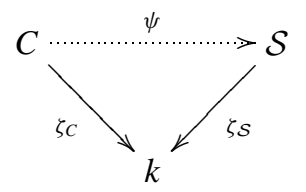

commutes.

Proof. We define $\psi$ by an explicit formula. To state it, we introduce some notation.

First, for each $n \geq 0$, define $\psi_{n}: C \rightarrow k$ by

$$
\psi_{n}(c)= \begin{cases}\zeta_{C}(c) & \text { if } c \in C_{n}, \\ 0 & \text { if not. }\end{cases}
$$

Second, for $\mathrm{S}=\left\{s_{1}<s_{2}<\cdots<s_{i}\right\} \subseteq\{1,2, \ldots, n\}$, let $s_{0}=0, s_{i+1}=n+1$ and

$$
\left(d_{1}, d_{2}, \ldots, d_{i+1}\right):=\left(s_{1}-s_{0}, s_{2}-s_{1}, \ldots, s_{i+1}-s_{i}\right)-(1,1, \ldots, 1) \in \mathbb{N}^{i+1} .
$$

Finally, define $\psi_{\mathrm{S}}: C^{\otimes(i+1)} \rightarrow k$ by $\psi_{\mathrm{S}}=\psi_{d_{1}} \otimes \psi_{d_{2}} \otimes \cdots \otimes \psi_{d_{i+1}}$. Then $\psi: C \rightarrow \mathcal{S}$ is defined on $c \in C_{n}$ by

$$
\psi(c)=\sum_{\mathrm{S} \in \mathcal{S}_{n}} \psi_{\mathrm{S}} \Delta^{(\# \mathrm{~S})}(c) \cdot \mathrm{S} .
$$

We first check that the diagram commutes. Take $c \in C_{n}$. Since $\zeta_{\mathcal{S}}$ annihilates all nonempty subsets of $\{1, \ldots, n\}$,

$$
\zeta_{\mathcal{S}} \psi(c)=\psi_{\emptyset} \Delta^{(0)}(c)=\psi_{n}(c)=\zeta_{C}(c),
$$

as needed. 
By definition, $\psi$ is degree preserving. Thus, to show that it is a morphism of graded coalgebras it only remains to verify that $\Delta \psi=(\psi \otimes \psi) \Delta$. The proof is based on the following facts:

(1) For any $n \geq 0$, there is a bijection $\left\{(\mathrm{S}, k) / \mathrm{S} \in \mathcal{S}_{n}, k \in \mathrm{S}\right\} \rightarrow \coprod_{k=1}^{n} \mathcal{S}_{k-1} \times \mathcal{S}_{n-k}$, given by

$$
\begin{aligned}
& (\mathrm{S}, k) \mapsto(\mathrm{S}(0, k), \mathrm{S}(k, n+1)) \in \mathcal{S}_{k-1} \times \mathcal{S}_{n-k} \text { and } \\
& (\mathrm{R}, \mathrm{T}) \in \mathcal{S}_{k-1} \times \mathcal{S}_{n-k} \mapsto(\mathrm{R} \cup\{k\} \cup(k+\mathrm{T}), k)
\end{aligned}
$$

(2) $\psi_{\mathrm{R} \cup\{k\} \cup(k+\mathrm{T})}=\psi_{\mathrm{R}} \otimes \psi_{\mathrm{T}}$ and $\Delta^{(\# \mathrm{R}+1+\# T)}=\Delta^{(\# \mathrm{R})} \otimes \Delta^{(\# \mathrm{~T})} \cdot \Delta$.

(3) If $\mathrm{S} \in \mathcal{S}_{n}, c \in C_{m}$ and $n \neq m$, then $\psi_{\mathrm{S}} \Delta^{(\# \mathrm{~S})}(c)=0$.

Assertions (1) and (2) are straightforward. In order to verify (3), write $\Delta^{(\# S)}(c)=$ $\sum c_{1} \otimes c_{2} \otimes \cdots \otimes c_{i+1}$. Then

$$
\psi_{\mathrm{S}} \Delta^{(\# \mathrm{~S})}(c)=\sum \psi_{d_{1}}\left(c_{1}\right) \otimes \psi_{d_{2}}\left(c_{2}\right) \cdots \psi_{d_{i+1}}\left(c_{i+1}\right) .
$$

Suppose this sum is not zero. Then some term must be nonzero. Hence, for this term, the degree of each $c_{j}$ equals $d_{j}=s_{j}-s_{j-1}-1$ (otherwise $c_{j}$ would be annihilated by $\psi_{d_{j}}$ ). Since $\Delta$ is homogeneous, it follows that the total degree of $\Delta^{(\# \mathrm{~S})}(c)$ is

$$
\sum_{j=1}^{i+1}\left(s_{j}-s_{j-1}-1\right)=s_{i+1}-s_{0}-(i+1)=n+1-(i+1)=n-i .
$$

On the other hand, since $\Delta$ decreases degrees by one, this total degree must also be equal to the degree of $c$ minus \#S, i.e. $m-i$. We conclude that $n=m$. This proves assertion (3).

The proof proceeds as follows. Take $c \in C_{n}$ and write $\Delta(c)=\sum_{j=1}^{n} c_{j-1} \otimes c_{n-j}^{\prime}$, with $c_{j-1} \in C_{j-1}$ and $c_{n-j}^{\prime} \in C_{n-j}$. We have

$$
\begin{aligned}
\Delta \psi(c) & =\sum_{\mathrm{S} \in \mathcal{S}_{n}} \sum_{k \in \mathrm{S}} \psi_{\mathrm{S}} \Delta^{(\# \mathrm{~S})}(c) \mathrm{S}(0, k) \otimes \mathrm{S}(k, n+1) \\
& \stackrel{(1)}{=} \sum_{k=1}^{n} \sum_{\substack{\mathrm{R} \in \mathcal{S}_{k-1} \\
\mathrm{~T} \in \mathcal{S}_{n-k}}} \psi_{\mathrm{R} \cup\{k\} \cup(k+\mathrm{T})} \Delta^{(\# \mathrm{R}+1+\# T)}(c) \cdot \mathrm{R} \otimes \mathrm{T} \\
& \stackrel{(2)}{=} \sum_{k=1}^{n} \sum_{\substack{\mathrm{R} \in \mathcal{S}_{k-1} \\
\in \in \mathcal{S}_{n-k}}} \sum_{j=1}^{n} \psi_{\mathrm{R}} \Delta^{(\# \mathrm{R})}\left(c_{j-1}\right) \psi_{\mathrm{T}} \Delta^{(\# \mathrm{~T})}\left(c_{n-j}^{\prime}\right) \cdot \mathrm{R} \otimes \mathrm{T} \\
& \stackrel{(3)}{=} \sum_{k=1}^{n}\left(\sum_{\mathrm{R} \in \mathcal{S}_{k-1}} \psi_{\mathrm{R}} \Delta^{(\# \mathrm{R})}\left(c_{k-1}\right) \mathrm{R}\right) \otimes\left(\sum_{\mathrm{T} \in \mathcal{S}_{n-k}} \psi_{\mathrm{T}} \Delta^{(\# \mathrm{~T})}\left(c_{n-k}^{\prime}\right) \mathrm{T}\right) \\
& =\sum_{k=1}^{n} \psi\left(c_{k-1}\right) \otimes \psi\left(c_{n-k}^{\prime}\right)=(\psi \otimes \psi) \Delta(c)
\end{aligned}
$$

as needed. 
It only remains to verify uniqueness. Let $\varphi: C \rightarrow \mathcal{S}$ be another morphism of graded coalgebras such that $\zeta_{\mathcal{S}} \varphi=\zeta_{C}$. We show by induction on $n$ that $\psi$ and $\varphi$ agree on $C_{n}$.

For $c \in C_{0}$, we must have $\varphi(c)=\lambda \emptyset_{0}$ for some $\lambda \in k$, since $\varphi$ is degree preserving. Then

$$
\lambda=\zeta_{\mathcal{S}} \varphi(c)=\zeta_{C}(c)=\psi_{0}(c)
$$

and thus

$$
\varphi(c)=\psi_{0}(c) \emptyset_{0}=\psi(c) .
$$

Assume now that $\psi$ and $\varphi$ agree on $C_{i}$ for every $i<n$. Since $\Delta$ decreases degrees, we can assert that for any $c \in C_{n}$,

$$
\Delta \varphi(c)=(\varphi \otimes \varphi) \Delta(c)=(\psi \otimes \psi) \Delta(c)=\Delta \psi(c) .
$$

In other words, $\psi(c)-\varphi(c) \in \operatorname{Ker}\left(\Delta_{\left.\right|_{\mathcal{S}_{n}}}\right)$. Now, from the definition of the comultiplication of $\mathcal{S}$ it is clear that $\operatorname{Ker}\left(\Delta_{\mathcal{S}_{n}}\right)$ is the one-dimensional space spanned by $\emptyset_{n}$. Therefore,

$$
\psi(c)-\varphi(c)=\lambda \emptyset_{n}
$$

for some new scalar $\lambda \in k$. Applying $\zeta_{\mathcal{S}}$ we obtain $\lambda=0$. Thus $\psi(c)=\varphi(c)$ for every $c \in C_{n}$. The proof is complete.

The following are the first values of $\psi$, according to its explicit definition 4.2:

$$
\begin{array}{rlrl}
\text { if } \quad c \in C_{0}, & \psi(c) & =\psi_{0}(c) \cdot \emptyset_{0} ; \\
\text { if } \quad c \in C_{1}, & \psi(c)= & \psi_{1}(c) \cdot \emptyset_{1}+\left(\psi_{0} \otimes \psi_{0}\right) \Delta(c) \cdot\{1\}_{1} ; \\
\text { if } \quad c \in C_{2}, & \psi(c)= & \psi_{2}(c) \cdot \emptyset_{2}+\left(\psi_{0} \otimes \psi_{1}\right) \Delta(c) \cdot\{1\}_{2}+\left(\psi_{1} \otimes \psi_{0}\right) \Delta(c) \cdot\{2\}_{2} \\
& & & +\left(\psi_{0} \otimes \psi_{0} \otimes \psi_{0}\right) \Delta
\end{array}
$$

The universal property of the algebra of sets admits a few variants, which are useful for the study of the cd-index. The first of these involves comodules and coderivations. Recall that, if $(C, \Delta)$ is a coalgebra, a $C$-bicomodule consists of a triple $(M, s, t)$ where $M$ is a space and $s: M \rightarrow M \otimes C$ and $t: M \rightarrow C \otimes M$ are linear maps such that the following diagrams commute:
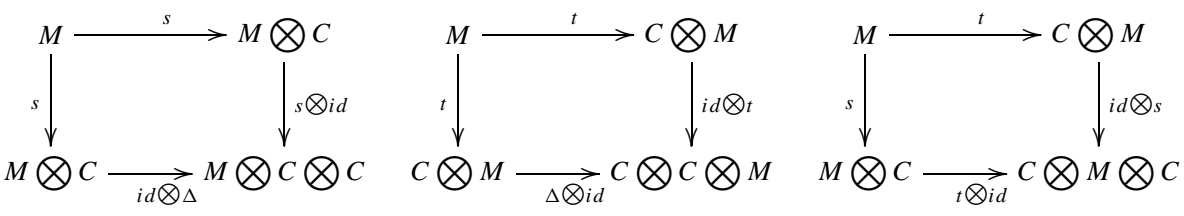

For instance, $(C, \Delta, \Delta)$ and $(C \otimes C, i d \otimes \Delta, \Delta \otimes i d)$ are $C$-bicomodules.

A coderivation is a map $\delta: M \rightarrow C$ such that

$$
\Delta \delta=(\delta \otimes i d) s+(i d \otimes \delta) t .
$$

If $C$ is graded, then we say that $M$ is a graded bicomodule if there is given a decomposition $M=\bigoplus_{n=0}^{\infty} M_{n}$ with $s\left(M_{n}\right) \subseteq \sum_{i=1}^{n} M_{i-1} \otimes C_{n-i}$ and $t\left(M_{n}\right) \subseteq \sum_{i=1}^{n} C_{i-1} \bigotimes$ $M_{n-i}$. 
Corollary 4.2. Let $M$ be a graded $\mathcal{S}$-bicomodule and let $\zeta_{M}: M \rightarrow k$ be a linear functional. Then there is a unique degree-preserving coderivation $\delta: M \rightarrow \mathcal{S}$ such that

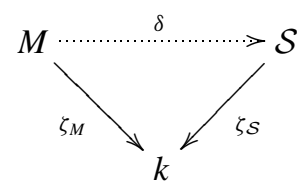

commutes.

Proof. It is possible to give a direct proof, similar to the proof of Theorem 4.1. Alternatively, one may resort to the standard trick of viewing coderivations as morphisms of coalgebras, and apply Theorem 4.1. We leave the details to the reader.

Corollary 4.3. Let A be a graded E-bialgebra (in the sense of (2.7)) and let $\zeta_{A}: A \rightarrow k$ be a morphism of algebras. Then there exists a unique morphism of graded $\varepsilon$-Hopf algebras $\psi: A \rightarrow \mathcal{S}$ such that

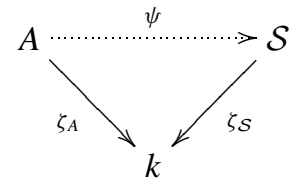

commutes.

Proof. According to Theorem 4.1, there is a unique morphism of graded coalgebras $\psi: A \rightarrow \mathcal{S}$ such that $\zeta_{\mathcal{S}} \psi=\zeta_{A}$. We only have to show that, under the present hypothesis, $\psi$ is also a morphism of algebras, i.e. that $\psi m_{A}=m_{\mathcal{S}}(\psi \otimes \psi)$. (Recall that a morphism of $\varepsilon$-bialgebras always preserves the antipodes.)

First, note that since $\zeta_{A}$ and $\zeta_{\mathcal{S}}$ are morphisms of algebras, the following diagrams commute:
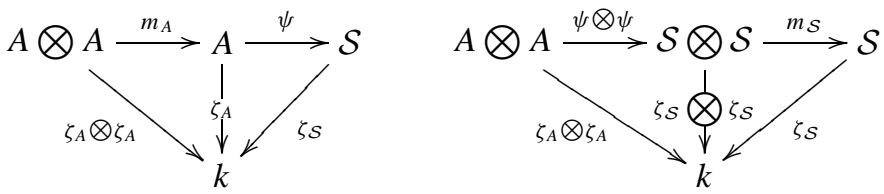

We appeal to the uniqueness in Corollary 4.2. Since $A$ is a graded $\varepsilon$-bialgebra, $m_{A}$ is a degree-preserving coderivation, and since $\psi$ is a morphism of graded coalgebras, $\psi m_{A}: A \otimes A \rightarrow \mathcal{S}$ is a degree-preserving coderivation. Similarly, since $m_{\mathcal{S}}$ is a degreepreserving coderivation and $\psi \otimes \psi$ is a degree-preserving morphism of $\mathcal{S}$-bicomodules, $m_{\mathcal{S}}(\psi \otimes \psi)$ is a degree-preserving coderivation. Therefore, by uniqueness of degreepreserving coderivations into $\mathcal{S}$ (Corollary 4.2), the composites along the top of the diagrams above must coincide. This is the desired conclusion. 
Remark. In the situation of Corollary 4.3, suppose that $A$ possesses a unit element $1 \in A_{0}$ and that $\zeta_{A}$ preserves units. Then

$$
\psi(1)=\psi_{0}(1) \emptyset_{0}=\zeta_{A}(1) \emptyset_{0}=\emptyset_{0},
$$

so $\psi$ preserves units as well.

Example 4.4. We apply the universal property of the algebra of sets to the algebra of posets and the functional $\zeta_{\mathcal{P}}: \mathcal{P} \rightarrow k$ defined in Section 3. Since $\zeta_{\mathcal{P}}$ is a morphism of algebras, Corollary 4.3 yields a unique morphism of $\varepsilon$-Hopf algebras $\psi: \mathcal{P} \rightarrow \mathcal{S}$ such that $\zeta_{\mathcal{S}} \psi=\zeta_{\mathcal{P}}$. It follows readily from (4.2) that this is none other than the ab-index of posets. We work out the details of this calculation in Section 5.2, after introducing the necessary notation.

\section{Applications of the Universal Property. The ab-Index of Posets}

In this section we derive some important applications of the universal property, including the construction of the ab-index of posets as a morphism of $\varepsilon$-Hopf algebras and also of several variants of it. In particular, we define a version of the ab-index for weighted posets, that generalizes not only the usual ab-index but also several other versions in the literature, as the relative ab-index of Stanley.

Only the results from Sections 5.1 and 5.2 are needed for the construction of the cd-index in Section 6.

\subsection{The Algebra of Noncommutative Polynomials}

Consider the $\varepsilon$-Hopf algebra of Example 2.3(1), i.e. the algebra $k\langle\mathbf{a}, \mathbf{b}\rangle$ of noncommutative polynomials with

$$
\Delta(\mathbf{a})=\Delta(\mathbf{b})=1 \otimes 1
$$

Corollary 5.1. There is an isomorphism of graded $\varepsilon$-Hopf algebras $\mathcal{S} \rightarrow k\langle\mathbf{a}, \mathbf{b}\rangle$ sending $\mathrm{S} \in \mathcal{S}_{n}$ to $u_{\mathrm{S}}=u_{1} u_{2} \cdots u_{n}$, where

$$
u_{i}= \begin{cases}\mathbf{a}-\mathbf{b} & \text { if } i \notin \mathrm{S} \\ \mathbf{b} & \text { if } i \in \mathrm{S} .\end{cases}
$$

Proof. Define a morphism of algebras $\zeta_{1,0}: k\langle\mathbf{a}, \mathbf{b}\rangle \rightarrow k$ by

$$
\zeta_{1,0}(p(\mathbf{a}, \mathbf{b}))=p(1,0) .
$$

By the universal property of $\mathcal{S}$ (Corollary 4.3), there exists a unique morphism of graded $\mathcal{E}$-Hopf algebras $\psi: k\langle\mathbf{a}, \mathbf{b}\rangle \rightarrow \mathcal{S}$ such that $\zeta_{\mathcal{S}} \psi=\zeta_{1,0}$. From the explicit formula (4.1) 
for $\psi$ we see that

$$
\begin{aligned}
\psi(\mathbf{a}) & =\psi_{1}(\mathbf{a}) \cdot \emptyset_{1}+\left(\psi_{0} \otimes \psi_{0}\right) \Delta(\mathbf{a}) \cdot\{1\}_{1} \\
& =\zeta_{1,0}(\mathbf{a}) \cdot \emptyset_{1}+\zeta_{1,0}(1) \zeta_{1,0}(1) \cdot\{1\}_{1}=\emptyset_{1}+\{1\}_{1}, \\
\psi(\mathbf{b}) & =\psi_{1}(\mathbf{b}) \cdot \emptyset_{1}+\left(\psi_{0} \otimes \psi_{0}\right) \Delta(\mathbf{b}) \cdot\{1\}_{1} \\
& =\zeta_{1,0}(\mathbf{b}) \cdot \emptyset_{1}+\zeta_{1,0}(1) \zeta_{1,0}(1) \cdot\{1\}_{1}=\{1\}_{1} .
\end{aligned}
$$

Hence,

$$
\psi\left(u_{i}\right)= \begin{cases}\emptyset_{1} & \text { if } \quad i \notin \mathrm{S}, \\ \{1\}_{1} & \text { if } \quad i \in \mathrm{S} .\end{cases}
$$

It follows immediately from the definition of multiplication in $\mathcal{S}$ that

$$
\psi\left(u_{\mathrm{S}}\right)=\psi\left(u_{1}\right) \psi\left(u_{2}\right) \cdots \psi\left(u_{n}\right)=\mathrm{S}, \quad \forall \mathrm{S} \in \mathcal{S}_{n} .
$$

In particular, $\psi: k\langle\mathbf{a}, \mathbf{b}\rangle_{n} \rightarrow \mathcal{S}_{n}$ is surjective. Since $\operatorname{dim} k\langle\mathbf{a}, \mathbf{b}\rangle_{n}=2^{n}=\operatorname{dim} \mathcal{S}_{n}$, it follows that $\psi: k\langle\mathbf{a}, \mathbf{b}\rangle \rightarrow \mathcal{S}$ is an isomorphism.

Remark. It is easy to see that the universal property of the coalgebra $\mathcal{S}$ (Theorem 4.1) may be equivalently expressed as follows: $\mathcal{S}$ is the cofree noncounital graded coalgebra on countably many cogenerators $\left\{\zeta_{n}\right\}_{n \geq 0}$, one cogenerator $\zeta_{n} \in\left(\mathcal{S}_{n}\right)^{*}$ of degree $n$ for each $n \geq 0$. Therefore, the graded dual of the coalgebra $\mathcal{S}$ is the free nonunital graded algebra on countably many generators, one generator for each degree $n \geq 0$. In view of the preceding corollary, this result applies to the graded dual of the coalgebra $k\langle\mathbf{a}, \mathbf{b}\rangle$. In this form, this result was obtained before by Billera and Liu [BL, Proposition 5.2].

The corollary allows us to apply the universal properties of Section 4 to the algebra $k\langle\mathbf{a}, \mathbf{b}\rangle$, equipped with the functional defined by (5.2). This amounts to replacing $\mathrm{S}$ for $u_{\mathrm{S}}$ in (4.1). We do this in a number of situations next.

\subsection{The ab-Index of Posets}

We apply the universal property of $k\langle\mathbf{a}, \mathbf{b}\rangle$ to the algebra of posets and the functional $\zeta_{\mathcal{P}}: \mathcal{P} \rightarrow k$ defined, as in Section 3 , by $\zeta_{\mathcal{P}}(P)=1$ for every poset $P$. Since $\zeta_{\mathcal{P}}$ is a morphism of algebras, Corollaries 4.3 and 5.1 yield a unique morphism of $\varepsilon$-Hopf algebras $\psi: \mathcal{P} \rightarrow k\langle\mathbf{a}, \mathbf{b}\rangle$ such that

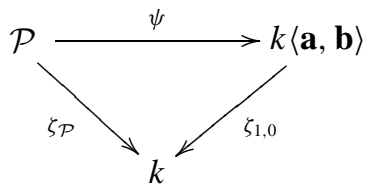


commutes. This is none other than the ab-index of posets. To see this, we calculate $\psi(P)$ using the explicit formula (4.1). First note that

$$
\psi_{n}(P)= \begin{cases}1 & \text { if } \operatorname{rank}(P)=n+1, \\ 0 & \text { if not. }\end{cases}
$$

Let $\mathrm{S}=\left\{s_{1}<s_{2}<\cdots<s_{i}\right\}$ be a subset of $\{1,2, \ldots, n\}, s_{0}=0, s_{i+1}=n+1$ and $d_{j}=s_{j}-s_{j-1}-1$ for $j=1, \ldots, i+1$. Then

$$
\begin{aligned}
& \psi_{\mathrm{S}} \Delta^{(i)}(P)= \sum_{0_{P}=x_{0}<x_{1}<x_{2}<\cdots<x_{i}<x_{i+1}=1_{P}} \psi_{d_{1}}\left(\left[0_{P}, x_{1}\right]\right) \psi_{d_{2}}\left(\left[x_{1}, x_{2}\right]\right) \cdots \psi_{d_{i+1}}\left(\left[x_{i}, 1\right]\right) \\
&= \#\left\{0_{P}<x_{1}<x_{2}<\cdots<x_{i}<1_{P} / \operatorname{rank}\left(\left[x_{j-1}, x_{j}\right]\right)=d_{j}+1,\right. \\
&\quad \forall j=1, \ldots, i+1\} \\
&=\#\left\{0_{P}<x_{1}<x_{2}<\cdots<x_{i}<1_{P} / \operatorname{rank}\left(x_{j}\right)=s_{j}, \forall j=1, \ldots, i\right\} .
\end{aligned}
$$

This number is usually called the flag number of $P$ corresponding to the set of ranks $S$, and it is denoted $f_{\mathrm{S}}(P)$. Thus, formula (4.1) gives

$$
\psi(P)=\sum_{\mathrm{S} \in \mathcal{S}_{n}} f_{\mathrm{S}}(P) \cdot u_{\mathrm{S}},
$$

which is the usual definition of the $\mathbf{a b}$-index of posets, see [BK] or [S2].

The fact that this formula defines a morphism of $\varepsilon$-bialgebras $\psi: \mathcal{P} \rightarrow k\langle\mathbf{a}, \mathbf{b}\rangle$ is known, and due to Ehrenborg and Readdy [ER, Proposition 3.1]. The approach taken in this work allows us to arrive at the ab-index from minimal data: the zeta function of posets.

\subsection{Automorphisms of $k\langle\mathbf{a}, \mathbf{b}\rangle$}

For any (graded) $\varepsilon$-Hopf algebra $A$, the set of (graded) automorphisms $\alpha: A \rightarrow A$ of $\varepsilon$-Hopf algebras forms a group under composition, denoted Aut $(A)$.

When the elements of an $\varepsilon$-Hopf algebra $A$ consist of combinatorial objects, as in the case $A=\mathcal{P}$ above, one may view a morphism of $\varepsilon$-Hopf algebras $A \rightarrow k\langle\mathbf{a}, \mathbf{b}\rangle$ as the association of a generating function (in two variables) to each combinatorial object of A. Clearly, two such morphisms will keep track of the same combinatorial information if they are related by an automorphism of $k\langle\mathbf{a}, \mathbf{b}\rangle$. For this reason, one would like a description of the group $\operatorname{Aut}(k\langle\mathbf{a}, \mathbf{b}\rangle)$. This can be easily obtained, thanks again to the universal property of $k\langle\mathbf{a}, \mathbf{b}\rangle$.

Recall that the group of affine transformations of the line is

$$
\operatorname{Aff}(k)=k \rtimes k^{\times}=\{(v, r) / v \in k, r \in k, r \neq 0\}
$$

with multiplication $(v, r) \cdot(w, s)=(v+r w, r s)$ and unit $(0,1)$. The group $\operatorname{Aff}(k)$ acts on the line $k$ by $(v, r) \cdot x=v+r x$. 
Claim. The group of automorphisms of the graded $\varepsilon$-Hopf algebra $k\langle\mathbf{a}, \mathbf{b}\rangle$ is the group of affine transformations of the line.

Proof. According to the universal property of $k\langle\mathbf{a}, \mathbf{b}\rangle$ (Corollary 4.3), an endomorphism $\alpha: k\langle\mathbf{a}, \mathbf{b}\rangle \rightarrow k\langle\mathbf{a}, \mathbf{b}\rangle$ of graded $\varepsilon$-Hopf algebras is uniquely determined by the morphism of algebras $\zeta_{1,0} \alpha: k\langle\mathbf{a}, \mathbf{b}\rangle \rightarrow k$. Since $k\langle\mathbf{a}, \mathbf{b}\rangle$ is free, such morphism is in turn uniquely determined by the pair of scalars

$$
q=\zeta_{1,0} \alpha(\mathbf{a}) \quad \text { and } \quad p=\zeta_{1,0} \alpha(\mathbf{b}) .
$$

Formula (4.1) gives

$$
\alpha(\mathbf{a})=q \mathbf{a}+(1-q) \mathbf{b} \quad \text { and } \quad \alpha(\mathbf{b})=p \mathbf{a}+(1-p) \mathbf{b} .
$$

Moreover, the universal property also guarantees that $\alpha$ is invertible if and only if it is invertible on the piece of degree 1 , i.e. if and only if $q \neq p$. Thus,

$$
\operatorname{Aut}(k\langle\mathbf{a}, \mathbf{b}\rangle) \rightarrow \operatorname{Aff}(k), \quad \alpha \mapsto(p, q-p)
$$

is a bijection. It is easy to see that this preserves the group structures.

The automorphism of $\mathcal{S}$ corresponding to $(v, r) \in \operatorname{Aff}(k)$ is given on $\mathrm{S} \in \mathcal{S}_{n}$ by

$$
\alpha(\mathrm{S})=r^{n-\# \mathrm{~S}} \sum_{\mathrm{T} \subseteq \mathrm{S}} v^{\# \mathrm{~S}-\# \mathrm{~T} T} \in \mathcal{S}_{n},
$$

as one readily sees by combining the previous result with Corollary 5.1.

\subsection{Edge Labelled Posets}

An edge labelled poset is a triple $(P, \lambda, L)$ where $P$ and $L$ are posets and $\lambda: H(P) \rightarrow L$ is an arbitrary assignment of an element of $L$ to each edge of the Hasse diagram of $P$,

$$
H(P)=\{(x, y) \in P \times P / x<y \text { and there is no } z \text { such that } x<z<y\} .
$$

We assume that $P$ is a finite, graded poset (as before), while $L$ may be an arbitrary poset. Two edge labelled posets $(P, \lambda, L)$ and $(Q, \mu, M)$ are isomorphic if there are isomorphisms of posets $P \cong Q$ and $L \cong M$ preserving the labellings $\lambda$ and $\mu$.

We define a new graded $\varepsilon$-Hopf algebra $\mathcal{P}_{E L}$, consisting of edge labelled posets. As a vector space, $\mathcal{P}_{E L}$ has a basis consisting of the isomorphism classes of edge labelled posets $(P, \lambda, L)$. The comultiplication is

$$
\Delta(P, \lambda, L)=\sum_{0_{P}<x<1_{P}}\left(\left[0_{P}, x\right], \lambda_{\left.\right|_{H\left(\left[O_{P}, x\right]\right)}}, L\right) \otimes\left(\left[x, 1_{P}\right], \lambda_{\left.\right|_{\left.H\left(x, 1_{P}\right]\right)}}, L\right) .
$$

To describe the multiplication, we first define a product $L * M$ of arbitrary posets as follows. We adjoin a top element to $L$ and a bottom element to $M$ and form the cartesian product of the resulting posets:

$$
L * M=\left(L \cup\left\{1_{L}\right\}\right) \times\left(\left\{0_{M}\right\} \cup M\right), \quad(l, m) \leq\left(l^{\prime}, m^{\prime}\right) \quad \Longleftrightarrow \quad l \leq l^{\prime} \quad \text { and } \quad m \leq m^{\prime} .
$$


Note that this is an associative operation. Now we define a multiplication in $\mathcal{P}_{E L}$ by

$$
(P, \lambda, L) \cdot(Q, \mu, M)=(P Q, \nu, L * M)
$$

where $P Q$ is defined as before (Example 2.3(2)), and $v: H(P Q) \rightarrow L * M$ is

$$
v(x, y)= \begin{cases}\left(\lambda(x, y), 0_{M}\right) & \text { if } \quad(x, y) \in H(P), \\ \left(1_{L}, \mu(x, y)\right) & \text { if } \quad(x, y) \in H(Q), \\ \left(\lambda\left(x, 1_{P}\right), \mu\left(0_{Q}, y\right)\right) & \text { if } \quad\left(x, 1_{P}\right) \in H(P) \quad \text { and } \quad\left(0_{Q}, y\right) \in H(Q) .\end{cases}
$$

By construction, the map $(P, \lambda, L) \mapsto P$ is a morphism of graded $\varepsilon$-Hopf algebras $\mathcal{P}_{E L} \rightarrow \mathcal{P}$.

Given a chain $0_{P}=x_{0}<x_{1}<\cdots<x_{n}<x_{n+1}=1_{P}$ in an edge labelled poset $(P, \lambda, L)$, a $\lambda$-descent of the chain is an index $i=1, \ldots, n$ such that

$$
\lambda\left(x_{i-1}, x_{i}\right) \not \leq \lambda\left(x_{i}, x_{i+1}\right) \quad \text { in } L .
$$

A chain is $\lambda$-increasing if it has no $\lambda$-descents, i.e. if $\lambda\left(x_{i-1}, x_{i}\right) \leq \lambda\left(x_{i}, x_{i+1}\right), \forall i$.

Consider the map $\zeta_{E L}: \mathcal{P}_{E L} \rightarrow k$ defined by

$$
\zeta_{E L}(P, \lambda, L)=\text { the number of } \lambda \text {-increasing maximal chains in } P \text {. }
$$

It is easy to see that $\zeta_{E L}$ is a morphism of algebras. Therefore, by the universal property of $k\langle\mathbf{a}, \mathbf{b}\rangle$ (Corollary 4.3), there is a unique morphism of graded $\varepsilon$-Hopf algebras $\psi_{E L}: \mathcal{P}_{E L} \rightarrow k\langle\mathbf{a}, \mathbf{b}\rangle$ such that $\zeta_{1,0} \psi_{E L}=\zeta_{E L}$. Working out formula 4.2 as in Section 5.2 , one readily finds that $\psi_{E L}$ is given on a poset $P$ of rank $n+1$ by

$$
\psi_{E L}(P, \lambda, L)=\sum_{\mathrm{S} \in \mathcal{S}_{n}} f_{\mathrm{S}}(P, \lambda, L) \cdot u_{\mathrm{S}}
$$

where $f_{\mathrm{S}}(P, \lambda, L)$ is the number of maximal chains in $P$ whose $\lambda$-descents are contained in $\mathrm{S}$. One may view the morphism $\psi_{E L}$ as a version of the ab-index specific to edge labelled posets. As in the case of the usual ab-index, it is easy to see directly that this formula indeed defines a morphism of $\varepsilon$-Hopf algebras.

Consider now those labelled posets $(P, \lambda, L)$ for which every interval contains exactly one $\lambda$-increasing maximal chain. Such posets are called $R$-labelled. Since this condition refers to all intervals of the poset, $R$-labelled posets span a subcoalgebra of $\mathcal{P}_{E L}$, that we denote by $\mathcal{P}_{R L}$. Clearly, $\mathcal{P}_{R L}$ is also an $\varepsilon$-Hopf subalgebra of $\mathcal{P}_{E L}$.

Now, by definition, if $(P, \lambda, L) \in \mathcal{P}_{R L}$, then

$$
\zeta_{E L}(P, \lambda, L)=1=\zeta_{\mathcal{P}}(P) .
$$

It follows, by uniqueness in the universal property of $k\langle\mathbf{a}, \mathbf{b}\rangle$ applied to the $\varepsilon$-Hopf algebra $\mathcal{P}_{R L}$, that for every $(P, \lambda, L) \in \mathcal{P}_{R L}$,

$$
\psi_{E L}(P, \lambda, L)=\psi(P) .
$$

Thus, if $(P, \lambda, L)$ is an $R$-labelled poset of rank $n+1$, then

$$
f_{\mathrm{S}}(P, \lambda, L)=f_{\mathrm{S}}(P)
$$

for any $S \subseteq\{1, \ldots, n\}$. This equality has been noted by Björner [B, Theorem 2.7] (again, it is very easy to give a direct proof). 
Remark. An equivalent version of the ab-index of edge labelled posets $\psi_{E L}$ was constructed by Bergeron and Sottile [BS], from the point of view of ordinary Hopf algebras. In this reference, and often in the literature, the labellings take values on totally ordered sets. In order to obtain an $\varepsilon$-Hopf algebra, one has to allow poset-valued labellings, as done above. We thank the referee for pointing this out to us.

\subsection{The ab-Index of Weighted Posets}

As a final application of the universal property, we construct a very natural version of the $\mathbf{a b}$-index that generalizes at the same time the usual $\mathbf{a b}$-index of posets, the $\mathbf{a b}$-index of edge labelled posets of Section 5.4 and also the relative ab-index of Stanley.

A weight on a poset $P$ is an arbitrary scalar-valued map $w$ defined on the collection of nonempty intervals of $P$. Such a weight induces a weight on every interval of $P$, by restriction.

As before, we may construct an $\varepsilon$-bialgebra $\mathcal{P}_{W}$ consisting of pairs $(P, w)$, of (isomorphism classes of finite, graded) weighted posets. Explicitly, the comultiplication is

$$
\Delta(P, w)=\sum_{0_{P}<x<1_{P}}\left(\left[0_{P}, x\right], w_{\mid\left[o_{P}, x\right]}\right) \otimes\left(\left[x, 1_{P}\right], w_{\left[x, 1_{P}\right]}\right)
$$

and the multiplication is

$$
(P, u) \cdot(Q, v)=(P Q, w),
$$

where $P Q$ is defined as before (Example 2.3(2)) and

$$
w([x, y])= \begin{cases}u([x, y]) & \text { if } \quad 0_{P} \leq x<y<1_{P}, \\ v([x, y]) & \text { if } \quad 0_{Q}<x<y \leq 1_{P}, \\ u\left(\left[x, 1_{P}\right]\right) v\left(\left[0_{Q}, y\right]\right) & \text { if } x<1_{P}, \quad 0_{Q}<y .\end{cases}
$$

The $\varepsilon$-bialgebra $\mathcal{P}_{W}$ comes equipped with a canonical morphism of algebras $\zeta_{W}: \mathcal{P}_{W} \rightarrow$ $k$ defined by

$$
\zeta_{W}(P, w)=w\left(\left[0_{P}, 1_{P}\right]\right)
$$

Hence, the universal property of $k\langle\mathbf{a}, \mathbf{b}\rangle$ yields a unique morphism of $\varepsilon$-bialgebras $\psi_{W}: \mathcal{P}_{W} \rightarrow k\langle\mathbf{a}, \mathbf{b}\rangle$ such that $\zeta_{1,0} \psi_{W}=\zeta_{W}$. Formula (4.1) immediately gives, for a graded poset $P$ of rank $n+1$ with weight $w$,

$$
\psi_{W}(P, w)=\sum_{\mathrm{S} \in \mathcal{S}_{n}} f_{\mathrm{S}}(P, w) \cdot u_{\mathrm{S}}
$$

where, for $\mathrm{S}=\left\{s_{1}<s_{2}<\cdots<s_{i}\right\} \subseteq\{1,2, \ldots, n\}$,

$$
f_{\mathrm{S}}(P, w)=\sum_{\substack{0 \\ 0=x_{0}<x_{1}<x_{2}<\cdots<x_{i}<x_{i+1}=1 \\ \operatorname{rank}\left(x_{i}\right)=s_{i}}} w\left(\left[0_{P}, x_{1}\right]\right) w\left(\left[x_{1}, x_{2}\right]\right) \cdots w\left(\left[x_{i}, 1\right]\right) .
$$

We call $\psi_{W}$ the ab-index of weighted posets. It generalizes several versions of the abindex, as we now explain. 
First, any poset $P$ carries the trivial weight $e$ defined by $e([x, y])=1$ for any $x<y$ in $P$. This defines a morphism of $\varepsilon$-bialgebras $\mathcal{P} \rightarrow \mathcal{P}_{W}, P \mapsto(P, e)$, which preserves the zeta functions, and hence also the ab-indices $\psi$ and $\psi_{W}$, by uniqueness. In other words, $f_{\mathrm{S}}(P, e)=f_{\mathrm{S}}(P)$.

To relate the ab-index of weighted posets to the ab-index of edge labelled posets, consider the map

$$
\mathcal{P}_{E L} \rightarrow \mathcal{P}_{W}, \quad(P, \lambda, L) \mapsto\left(P, w_{\lambda}\right),
$$

where

$$
w_{\lambda}([x, y])=\text { the number of } \lambda \text {-increasing maximal chains in }[x, y] \text {. }
$$

This is a morphism of $\varepsilon$-bialgebras which preserves the zeta functions $\zeta_{E L}$ and $\zeta_{W}$, and hence also the ab-indices $\psi_{E L}$ and $\psi_{W}$. We deduce (the simple fact) that the number of descents and the number of weighted chains are related by

$$
f_{\mathrm{S}}(P, \lambda, L)=f_{\mathrm{S}}\left(P, w_{\lambda}\right),
$$

for any edge labelled poset $(P, \lambda, L)$. If $(P, \lambda, L)$ is $R$-labelled, then $w_{\lambda}$ is the trivial weight, and we recover the result of Björner mentioned at the end of Section 5.4.

Finally, we indicate how the ab-index of weighted posets generalizes the relative ab-index of Stanley. To every collection $X$ of nontrivial intervals of $P$ one may associate a weight $w_{X}$ on $P$ defined by

$$
w_{X}([x, y])= \begin{cases}1 & \text { if }[x, y] \in X \\ 0 & \text { if not. }\end{cases}
$$

Formula (5.2) becomes

$$
\begin{array}{r}
f_{\mathrm{S}}\left(P, w_{X}\right)=\#\left\{0_{P}=x_{0}<x_{1}<\cdots<x_{i}<x_{i+1}=1_{P} / \operatorname{rank}\left(x_{j}\right)=s_{j}\right. \\
\text { and } \left.\left[x_{j-1}, x_{j}\right] \in X, \forall j\right\} .
\end{array}
$$

This is the relative flag vector of Section 1 of [S3].

Let $(P, \lambda, L)$ be an edge labelled poset for which every interval possesses at most one $\lambda$-increasing maximal chain. One may call such posets weakly $R$-labelled. In this case, the weight associated to the labelling coincides with the weight associated to the collection $X_{\lambda}$ of intervals which possess a $\lambda$-increasing maximal chain. Therefore, $(5.3)$ becomes

$$
\begin{array}{r}
f_{S}(P, \lambda, L)=\#\left\{0_{P}=x_{0}<x_{1}<\cdots<x_{i}<x_{i+1}=1_{P} / \operatorname{rank}\left(x_{j}\right)=s_{j}\right. \\
\text { and }\left[x_{j-1}, x_{j}\right] \text { possesses a } \lambda \text {-increasing maximal } \\
\text { chain, } \forall j\} .
\end{array}
$$

This is Theorem 4.4.a in [S3].

Remark. Stanley defines the relative flag vector from the set $\Gamma_{X}$ consisting of those chains $0_{P}<x_{1}<\cdots<x_{i}<1_{P}$ for which some interval $\left[x_{j-1}, x_{j}\right]$ is not in $X$ [S3, page 
5]. He assumes that the collection $X$ is closed under subintervals in order to guarantee that $\Gamma_{X}$ is a subcomplex of the simplicial complex of all chains in $P$. For the same reason, he makes an additional assumption on the edge labellings. These assumptions are not needed for the construction of a well-behaved ab-index , or to obtain (5.4), as we have seen.

\section{Eulerian Subalgebras. The cd-Index of Eulerian Posets}

In Section 5.2 we saw that to construct the ab-index of posets one only needs to know the zeta function of posets $\zeta: \mathcal{P} \rightarrow k$. The universal property of $k\langle\mathbf{a}, \mathbf{b}\rangle$ took care of the rest. Notice that this property only involves the $\varepsilon$-bialgebra structure of $\mathcal{P}$.

Similarly, to construct the cd-index of eulerian posets, all one needs is knowledge of the Möbius function of posets, $\mu: \mathcal{P} \rightarrow k$. As we saw in Section 3, the Möbius function is closely related to the antipode of the $\varepsilon$-Hopf algebra $\mathcal{P}$. It is the general notion of antipode that allows us to define a notion of "Möbius function" for arbitrary $\varepsilon$-Hopf algebras $A$ equipped with a morphism of algebras $\zeta: A \rightarrow k$.

Definition 6.1. Let $A$ be an $\varepsilon$-Hopf algebra, with antipode $S$, and let $\zeta: A \rightarrow k$ be a morphism of algebras. The Möbius function of $(A, \zeta)$ is the functional

$$
\mu=\zeta S: A \rightarrow k \text {. }
$$

As recalled at the end of Section 2, it follows that $-\mu: A \rightarrow k$ is a morphism of algebras.

For instance, if $A=k\langle\mathbf{a}, \mathbf{b}\rangle$ and $\zeta$ is defined as in (5.2) by $\zeta(p(\mathbf{a}, \mathbf{b}))=p(1,0)$, since the antipode satisfies $S(p(\mathbf{a}, \mathbf{b}))=-p(\mathbf{a}-1, \mathbf{b}-1)$ (Example 2.3(1)), the Möbius function satisfies $\mu(p(\mathbf{a}, \mathbf{b}))=-p(0,-1)$.

Suppose now that $A$ is a graded $\varepsilon$-bialgebra and $\zeta: A \rightarrow k$ is a morphism of algebras. Then $A$ is automatically an $\varepsilon$-Hopf algebra, so the Möbius function $\mu$ of $(A, \zeta)$ is defined. Consider the functional $\alpha: A \rightarrow k$ defined on a homogeneous element $x \in A_{n}$ by

$$
\alpha(x)=\mu(x)+(-1)^{n} \zeta(x) .
$$

Observe that $\alpha$ satisfies, for $x \in A_{n}$ and $y \in A_{m}, \alpha(x y)=-\mu(x) \alpha(y)+(-1)^{n} \alpha(x) \zeta(y)$. Define a graded subspace of $A$ by

$$
E_{0}(\zeta)=\bigoplus_{n=0}^{\infty} \operatorname{Ker}\left(\alpha: A_{n} \rightarrow k\right)
$$

In view of the above, $E_{0}(\zeta)$ is a graded subalgebra of $A$.

Definition 6.2. Let $A$ be as above. The eulerian subalgebra of $(A, \zeta)$ is the largest subcoalgebra of $A$ contained in $E_{0}(\zeta)$. We denote it by $E(\zeta)$.

We will see below that $E(\zeta)$ is an $\varepsilon$-Hopf subalgebra of $A$, which justifies the terminology.

Given any coalgebra $C$ and a subspace $E_{0}$ of $C$, the largest subcoalgebra $E$ of $C$ contained in $E_{0}$ always exists: it is the sum of all subcoalgebras contained in $E_{0}$ (there 
is at least one, the zero subspace). In fact, this notion is dual to the notion of ideal generated by a subspace in an algebra. For this reason, one sometimes says that $E$ is the subcoalgebra of $C$ cogenerated by $E_{0}$. With this in mind, it is easy to obtain the following properties of eulerian subalgebras.

Proposition 6.3. Let $(A, \zeta)$ be as above and let $E(\zeta)$ be its eulerian subalgebra. Then:

(1) $E(\zeta)$ is a graded $\varepsilon$-Hopf subalgebra of $A$.

(2) The piece of degree $n$ of $E(\zeta)$ consists precisely of those $x \in A_{n}$ such that

$$
\begin{aligned}
\alpha(x) & =0, \\
(\alpha \bigotimes i d) \Delta(x) & =0=(i d \otimes \alpha) \Delta(x), \\
(i d \otimes \alpha \bigotimes i d) \Delta^{(2)}(x) & =0 .
\end{aligned}
$$

(3) Suppose that $\psi: A \rightarrow B$ is a morphism of graded $\varepsilon$-bialgebras such that

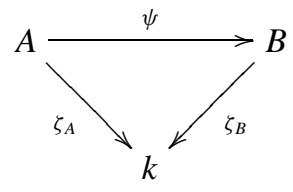

commutes. Then $\psi$ maps $E\left(\zeta_{A}\right)$ to $E\left(\zeta_{B}\right)$.

Proof. (1) We show, more generally, that the subcoalgebra cogenerated by a graded subalgebra of a graded $\varepsilon$-Hopf algebra is always a graded $\varepsilon$-Hopf subalgebra.

An ideal generated by homogeneous elements is necessarily graded. Dually, a subcoalgebra cogenerated by a graded subspace is necessarily graded. On the other hand, it follows immediately from (2.1) that if $C$ and $D$ are subcoalgebras of an $\varepsilon$-bialgebra $A$, then so is $C+D+C \cdot D$. Therefore, the subcoalgebra cogenerated by a subalgebra is again a subalgebra.

This shows that the subcoalgebra cogenerated by a graded subalgebra of a graded $\varepsilon$-bialgebra is always a graded $\varepsilon$-subbialgebra. Since by $(2.8)$ the antipode is determined by the bialgebra structure, the claim follows.

(2) Again, we provide a proof of a more general assertion. The ideal generated by a subspace $V$ of a nonunital algebra $(A, m)$ is

$$
V+m(V \otimes A)+m(A \bigotimes V)+m^{(2)}(A \bigotimes V \otimes A)
$$

(this subspace is closed under both left and right multiplications by associativity). If $V$ is defined as the image of a linear map $\alpha: A \rightarrow A$, then the ideal generated by $V$ is the sum of the images of the maps

$$
\alpha, m(\alpha \bigotimes i d), m(i d \otimes \alpha) \text { and } m^{(2)}(i d \otimes \alpha \bigotimes i d) .
$$

Dually, the subcoalgebra cogenerated by a subspace $V$ of a noncounital coalgebra $(C, \Delta)$ is

$$
V \cap \Delta^{-1}(C \otimes V) \cap \Delta^{-1}(V \otimes C) \cap \Delta^{(-2)}(C \otimes V \otimes C),
$$


and if $V$ is the kernel of a linear map $\alpha: C \rightarrow C$, then this can also be described as the intersection of the kernels of the maps

$$
\alpha,(\alpha \otimes i d) \Delta,(i d \otimes \alpha) \Delta \text { and }(i d \otimes \alpha \otimes i d) \Delta^{(2)} .
$$

With some care, these assertions can also be extended to the graded context, and the desired conclusion follows.

(3) By hypothesis $\psi$ preserves the zeta functionals, and hence also the alpha functionals, since a morphism of $\varepsilon$-bialgebras always preserves the antipodes. Therefore,

$$
\psi\left(E\left(\zeta_{A}\right)\right) \subseteq \psi\left(E_{0}\left(\zeta_{A}\right)\right) \subseteq E_{0}\left(\zeta_{B}\right)
$$

Since the image of a subcoalgebra under a morphism of coalgebras is another subcoalgebra, it follows that $\psi\left(E\left(\zeta_{A}\right)\right) \subseteq E\left(\zeta_{B}\right)$.

Recall that a graded poset $P$ is called eulerian if the Möbius function alternates between 1 and -1 on the intervals of $P$, or, more precisely, if for any $x \leq y$ in $P$,

$$
\mu_{\mathcal{P}}([x, y])=(-1)^{\operatorname{rank}(y)-\operatorname{rank}(x)} .
$$

The next is the main result of this work. It implies the existence of the cd-index of eulerian posets. Its value lies on the simplicity of the proof. For the second assertion, we assume that the characteristic of the field is not 2 .

Theorem 6.4. Consider the graded $\varepsilon$-Hopfalgebras $\mathcal{P}$ and $k\langle\mathbf{a}, \mathbf{b}\rangle$, with the functionals

$$
\zeta_{\mathcal{P}}: \mathcal{P} \rightarrow k, \quad \zeta_{\mathcal{P}}(P)=1 \quad \text { and } \quad \zeta_{1,0}: k\langle\mathbf{a}, \mathbf{b}\rangle \rightarrow k, \quad \zeta_{1,0}(p(\mathbf{a}, \mathbf{b}))=p(1,0)
$$

and the corresponding eulerian subalgebras. Then:

(1) $E\left(\zeta_{\mathcal{P}}\right)$ contains all eulerian posets.

(2) $E\left(\zeta_{1,0}\right)$ is the subalgebra of $k\langle\mathbf{a}, \mathbf{b}\rangle$ generated by $\mathbf{c}=\mathbf{a}+\mathbf{b}$ and $\mathbf{d}=\mathbf{a b}+\mathbf{b a}$.

Proof. (1) First, note that if a poset is eulerian, then so are any of its intervals. Hence, the class of eulerian posets spans a subcoalgebra of $\mathcal{P}$. Therefore, to show that the class of eulerian posets is contained in $E\left(\zeta_{\mathcal{P}}\right)$, it suffices to verify that it is contained in $E_{0}\left(\zeta_{\mathcal{P}}\right)$. This is immediate: if $P \in \mathcal{P}_{n}$ is an eulerian poset of rank $n+1$, then

$$
\alpha(P)=\mu_{\mathcal{P}}(P)+(-1)^{n} \zeta_{\mathcal{P}}(P) \stackrel{(6.2)}{=}(-1)^{n+1}+(-1)^{n}=0,
$$

so $P \in E_{0}\left(\zeta_{\mathcal{P}}\right)$.

Alternatively, one may verify (6.1) directly. Each of the four equations corresponds to the choice of an interval of $P$, which may be of the form $\left[0_{P}, 1_{P}\right],\left[0_{P}, x\right],\left[x, 1_{P}\right]$ or $[x, y]$.

(2) We abbreviate $E=E\left(\zeta_{1,0}\right)$ and $E_{0}=E_{0}\left(\zeta_{1,0}\right)$.

It is straightforward to verify (6.1) for $\mathbf{c}$ and $\mathbf{d}$. Since $E$ is a subalgebra, it follows that the subalgebra generated by $\mathbf{c}$ and $\mathbf{d}$ is contained in $E$. 
The converse inclusion requires a little work. Let $p(\mathbf{a}, \mathbf{b}) \in E$ be a homogeneous polynomial. Introduce a new variable $\mathbf{e}=\mathbf{a}-\mathbf{b}$. Then $\mathbf{c}$ and e generate $k\langle\mathbf{a}, \mathbf{b}\rangle$, so we can write

$$
p(\mathbf{a}, \mathbf{b})=\sum_{\mathbf{r}, \mathbf{s}} \lambda_{\mathbf{r s}} \mathbf{e}^{s_{0}} \mathbf{c}^{r_{1}} \mathbf{e}^{s_{1}} \mathbf{c}^{r_{2}} \cdots \mathbf{c}^{r_{n}} \mathbf{e}^{s_{n}} .
$$

This sum is over sequences of natural numbers $\mathbf{r}=\left(r_{1}, r_{2}, \ldots, r_{n}\right)$ and $\mathbf{s}=\left(s_{0}, s_{1}, \ldots\right.$, $\left.s_{n}\right)$, where only $s_{0}$ and $s_{n}$ are allowed to be zero, and $\lambda_{\text {rs }}$ are nonzero scalars.

Note that

$$
\mathbf{e}^{2}=(\mathbf{a}-\mathbf{b})^{2}=(\mathbf{a}+\mathbf{b})^{2}-2(\mathbf{a b}+\mathbf{b a})=\mathbf{c}^{2}-2 \mathbf{d} .
$$

Therefore, it suffices to show that all $s_{i}$ are even to conclude that $p(\mathbf{a}, \mathbf{b})$ belongs to the subalgebra generated by $\mathbf{c}$ and $\mathbf{d} .^{2}$

We verify this as follows. We have $\Delta(\mathbf{c})=2 \cdot 1 \otimes 1$ and $\Delta(\mathbf{e})=0$, and from here, using (2.1),

$$
\Delta^{(r)}\left(\mathbf{c}^{r}\right)=2^{r} \cdot \underbrace{1 \bigotimes \cdots \otimes 1}_{r+1} \text { and } \Delta\left(\mathbf{e}^{s}\right)=0 .
$$

Let $|\mathbf{r}|=r_{1}+r_{2}+\cdots+r_{n}$. It follows easily that

$$
\begin{gathered}
\Delta^{(|\mathbf{r}|)}\left(\mathbf{e}^{s_{0}} \mathbf{c}^{r_{1}} \mathbf{e}^{s_{1}} \mathbf{c}^{r_{2}} \cdots \mathbf{c}^{r_{n}} \mathbf{e}^{s_{n}}\right)=2^{|\mathbf{r}|} \mathbf{e}^{s_{0}} \bigotimes 1^{\otimes\left(r_{1}-1\right)} \otimes \mathbf{e}^{s_{1}} \otimes 1^{\otimes\left(r_{2}-1\right)} \bigotimes \cdots \\
\bigotimes 1^{\otimes\left(r_{n}-1\right)} \otimes \mathbf{e}^{s_{n}} \in A^{\otimes(|\mathbf{r}|+1)}
\end{gathered}
$$

Since $E$ is a subcoalgebra, $\Delta^{(|\mathbf{r}|)}(p(\mathbf{a}, \mathbf{b}))$ belongs to $E^{\otimes(|\mathbf{r}|+1)}$.

On the other hand, by definition of eulerian subalgebra we have

$$
E^{\otimes(|\mathbf{r}|+1)} \subseteq E_{0}^{\otimes(|\mathbf{r}|+1)} \subseteq \operatorname{Ker}(\alpha)^{\otimes(|\mathbf{r}|+1)}=\bigcap_{t=0}^{|\mathbf{r}|} A^{\otimes(t)} \otimes \operatorname{Ker}(\alpha) \otimes A^{\otimes(|\mathbf{r}|-t)}
$$

Hence, for any $t$, applying $i d^{\otimes(t)} \otimes \alpha \bigotimes i d^{\otimes(|\mathbf{r}|-t)}$ to $\Delta^{(|\mathbf{r}|)}(p(\mathbf{a}, \mathbf{b}))$ we must get zero. Choosing appropriate values of $t$, we obtain from $(*)$ that

$$
\begin{aligned}
& 0=\sum_{\mathbf{r}, \mathbf{s}} \lambda_{\mathbf{r s}} 2^{|\mathbf{r}|} \mathbf{e}^{s_{0}} \otimes 1^{\otimes\left(r_{1}-1\right)} \otimes \mathbf{e}^{s_{1}} \otimes \cdots \otimes 1^{\otimes\left(r_{i}-1\right)} \alpha\left(\mathbf{e}^{s_{i}}\right) \otimes \cdots \otimes 1^{\otimes\left(r_{n}-1\right)} \otimes \mathbf{e}^{s_{n}}, \\
& \quad \text { for any } \quad i=0, \ldots, n .
\end{aligned}
$$

Since $p(\mathbf{a}, \mathbf{b})$ is homogeneous, all terms in this sum belong to distinct homogeneous components of $A^{\otimes(|\mathbf{r}|+1)}$. Hence, by linear independence, and since $\operatorname{char}(k) \neq 2$, we deduce that

$$
\alpha\left(\mathbf{e}^{s_{i}}\right)=0, \quad \forall i=0, \ldots, n .
$$

However,

$$
\alpha\left(\mathbf{e}^{s_{i}}\right)=\mu\left(\mathbf{e}^{s_{i}}\right)+(-1)^{s_{i}} \zeta\left(\mathbf{e}^{s_{i}}\right)=-1+(-1)^{s_{i}},
$$

so each $s_{i}$ must be even. The proof is complete.

2 This is the same trick used by Stanley in his proof of the existence of the cd-index [S2, Theorem 1.1]. 
Corollary 6.5. If $P$ is an eulerian poset, then its $\mathbf{a b - i n d e x ~ b e l o n g s ~ t o ~ t h e ~ s u b a l g e b r a ~}$ of $k\langle\mathbf{a}, \mathbf{b}\rangle$ generated by $\mathbf{c}$ and $\mathbf{d}$.

Proof. The ab-index was defined in Section 5.2 as the unique morphism $\psi$ of graded $\varepsilon$-bialgebras for which

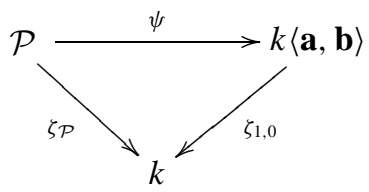

commutes. Therefore, by Proposition 6.3, $\psi$ takes the eulerian subalgebra of $\mathcal{P}$ to the eulerian subalgebra of $k\langle\mathbf{a}, \mathbf{b}\rangle$. Together with Theorem 6.4, this implies that if $P$ is an eulerian poset, then $\psi(P)$ belongs to the subalgebra generated by $\mathbf{c}$ and $\mathbf{d}$.

Remark. Ehrenborg and Readdy constructed an example of a non-eulerian poset $P$ for which $\psi(P)$ is nevertheless in the subalgebra generated by $\mathbf{c}$ and $\mathbf{d}$ [ER, Section 3]:

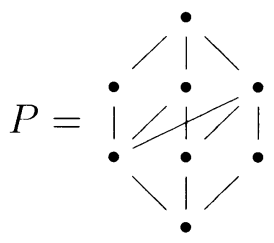

It turns out that the stronger fact that $P$ belongs to the eulerian subalgebra of $\mathcal{P}$ is also true. Thus, the eulerian subalgebra of $\mathcal{P}$ contains non-eulerian posets.

\section{The Generalized Dehn-Sommerville Equations}

Let $P$ be an eulerian poset. The fact that its ab-index $\psi(P)$ belongs to the subalgebra generated by $\mathbf{c}$ and $\mathbf{d}$ imposes some conditions on the coefficients of $\psi(P)$, i.e., on the flag vector $\left\{f_{\mathrm{S}}(P)\right\}_{\mathrm{S} \in \mathcal{S}_{n}}$. As recalled in the Introduction, according to Theorem 4 of $[\mathrm{BK}]$, these conditions are equivalent to the generalized Dehn-Sommerville equations of Bayer and Billera. We do not produce another proof of this fact here. Instead, we will provide another set of equations, equivalent to the equations of Bayer and Billera, that is dictated by the theory of $\varepsilon$-Hopf algebras, and is therefore very natural from the point of view of this work. As the equations of Bayer and Billera, our equations are indexed by triples $(\mathrm{S}, a, b)$ where $\mathrm{S}$ is a subset of $\{1, \ldots, n\}$ and $[a, b]$ is a maximal interval of $\{1, \ldots, n\} \backslash \mathrm{S}$. The equation corresponding to such a triple is

$$
\left((-1)^{b-a}+1\right) f_{\mathrm{S}}+\sum_{\mathrm{T} \subseteq[a, b], T \neq \emptyset}(-1)^{\# \mathrm{~T}} f_{\mathrm{S} \cup \mathrm{T}}=0 .
$$

To obtain these equations, we make use of the explicit description of eulerian subalgebras provided by (6.1), for the case of the $\varepsilon$-Hopf algebra $k\langle\mathbf{a}, \mathbf{b}\rangle$. 
It is convenient to deal with the algebra of sets $\mathcal{S}$ directly, rather than with the isomorphic algebra $k\langle\mathbf{a}, \mathbf{b}\rangle$. First, note that the zeta, Möbius and alpha functionals for this algebra are given on a basis element $S \in \mathcal{S}_{n}$ by

$$
\begin{aligned}
& \zeta(\mathrm{S})= \begin{cases}1 & \text { if } \mathrm{S}=\emptyset, \quad \mu(\mathrm{S})=(-1)^{\# \mathrm{~S}+1} \text { and } \\
0 & \text { if not, }\end{cases} \\
& \alpha(\mathrm{S})= \begin{cases}(-1)^{n}-1 & \text { if } \mathrm{S}=\emptyset_{n}, \\
(-1)^{\# \mathrm{~S}+1} & \text { if not. }\end{cases}
\end{aligned}
$$

Recall that in the $\varepsilon$-Hopf algebra of sets the comultiplication is

$$
\Delta(\mathrm{S})=\sum_{i \in \mathrm{S}} \mathrm{S}(0, i) \otimes \mathrm{S}(i, n+1)
$$

where $\mathrm{S}(i, j) \in \mathcal{S}_{j-i-1}$ consists of those elements of $\mathrm{S}$ that lie strictly between $i$ and $j$ (see the precise definition in Example 2.3(3)).

Let $x=\sum_{\mathrm{S} \in \mathcal{S}_{n}} f_{\mathrm{S}} \cdot \mathrm{S}$ be an arbitrary element of degree $n$ of $\mathcal{S}$. Let $\mathcal{T}_{n}$ denote the set of triples $(\mathrm{S}, a, b)$ as above, and for $(\mathrm{S}, a, b) \in \mathcal{T}_{n}$ let

$$
E_{\mathrm{S}, a, b}(x):=\left((-1)^{b-a-1}-1\right) f_{\mathrm{S}}-\sum_{\mathrm{T} \subseteq[a, b], T \neq \emptyset}(-1)^{\# \mathrm{~T}} f_{\mathrm{S} \cup \mathrm{T}} .
$$

Using the formulas above for $\alpha$ and $\Delta$ one finds, by direct calculations whose details we omit, that

$$
\begin{aligned}
\alpha(x) & =E_{\emptyset, 1, n}(x), \\
(\alpha \otimes i d) \Delta(x) & =\sum_{\substack{\mathrm{S} \in \mathcal{S}_{n} \\
\mathrm{~S} \neq 0,1 \notin \mathrm{S}}} E_{\mathrm{S}, 1, \min \mathrm{S}-1}(x) \cdot \mathrm{S}(\min \mathrm{S}, n+1), \\
(i d \otimes \alpha) \Delta(x) & =\sum_{\substack{\mathrm{S} \in \mathcal{S}_{n} \\
\mathrm{~S} \neq \emptyset, n \in \mathrm{S}}} E_{\mathrm{S}, \max \mathrm{S}+1, n}(x) \cdot \mathrm{S}(0, \max \mathrm{S}), \\
(i d \otimes \alpha \otimes i d) \Delta^{(2)}(x) & =\sum_{\substack{\mathrm{S}, a, b) \in \mathcal{T}_{n} \\
1<a, b<n}} E_{\mathrm{S}, a, b}(x) \cdot \mathrm{S}(0, a-1) \otimes \mathrm{S}(b+1, n+1) .
\end{aligned}
$$

These are expressions in terms of distinct basis elements of $\mathcal{S}$. In fact, suppose for instance that $\mathrm{S}(\min \mathrm{S}, n+1)=\mathrm{T}(\min \mathrm{T}, n+1)$ for two sets $\mathrm{S}$ and $\mathrm{T} \in \mathcal{S}_{n}$. Then, first, these elements must lie in the same homogeneous component of $\mathcal{S}$, which means that $\min S=\min T$. Second, the elements of $S$ lying strictly between $\min S$ and $n+1$ must coincide with the elements of T between $\min T$ and $n+1$. Thus, $\mathrm{S}=\mathrm{T}$. Similarly, suppose that $\mathrm{S}(0, a-1) \otimes \mathrm{S}(b+1, n+1)=\mathrm{T}(0, c-1) \otimes \mathrm{T}(d+1, n+1)$ for two triples $(\mathrm{S}, a, b)$ and $(\mathrm{T}, c, d) \in \mathcal{T}_{n}$. By comparing degrees, we see that $a=c$ and $b=d$. It then follows that the elements that lie strictly between 0 and $a-1$ or between $b+1$ and $n+1$ are the same for both $\mathrm{S}$ and T. Since, by definition of triple, the elements that lie between $a-1$ and $b+1$ are precisely $a-1$ and $b+1$, for both $\mathrm{S}$ and $\mathrm{T}$, we conclude that $\mathrm{S}=\mathrm{T}$.

Equations (6.1), defining eulerian subalgebras, express the vanishing of the above expressions. It follows that the element $x$ belongs to the eulerian subalgebra of $\mathcal{S}$ if and only if

$$
E_{\emptyset, 1, n}(x)=0,
$$




$$
\begin{aligned}
E_{\mathrm{S}, 1, \min \mathrm{S}-1}(x)=0 & \text { for every } \mathrm{S} \in \mathcal{S}_{n} \quad \text { such that } \mathrm{S} \neq \emptyset \text { and } 1 \notin \mathrm{S}, \\
E_{\mathrm{S}, \max \mathrm{S}+1, n}(x)=0 & \text { for every } \mathrm{S} \in \mathcal{S}_{n} \quad \text { such that } \mathrm{S} \neq \emptyset \text { and } n \notin \mathrm{S}, \\
E_{\mathrm{S}, a, b}(x)=0 & \text { for every triple } \quad(\mathrm{S}, a, b) \in \mathcal{T}_{n} \quad \text { such that } \\
& 1<a \text { and } b<n .
\end{aligned}
$$

Now, an arbitrary triple in $\mathcal{T}_{n}$ is of one the four forms above. Hence, these conditions can be expressed more simply as follows:

$$
E_{\mathrm{S}, a, b}(x)=0 \quad \text { for every triple } \quad(\mathrm{S}, a, b) \in \mathcal{T}_{n} .
$$

This is exactly the announced (7.1). It is the version of the generalized Dehn-Sommerville equations furnished by the theory of $\varepsilon$-Hopf algebras.

In conclusion, one may regard (6.1) as the generalized Dehn-Sommerville equations for an arbitrary $\varepsilon$-Hopf algebra. The main results of this work can be summarized as follows. The generalized Dehn-Sommerville equations for the $\varepsilon$-Hopf algebra $\mathcal{P}$ are satisfied by any eulerian poset. The generalized Dehn-Sommerville equations for the $\varepsilon$-Hopf algebra $k\langle\mathbf{a}, \mathbf{b}\rangle$ are explicitly given by (7.1), and they define the subalgebra generated by $\mathbf{c}$ and $\mathbf{d}$.

\section{References}

[A1] M. Aguiar, Infinitesimal Hopf algebras, Contem. Math. 267 (2000), 1-30.

[A2] M. Aguiar, On the associative analog of Lie bialgebras, to appear in J. Algebra.

[AF] M. Aguiar and W. Ferrer Santos, Galois connections for incidence Hopf algebras of partially ordered sets, Adv. Math. 151 (2000), 71-100.

[B] A. Björner, Shellable and Cohen-Macaulay partially ordered sets, Trans. Amer. Math. Soc. 260 (1980), 159-183.

[BB] M. Bayer and L. Billera, Generalized Dehn-Sommerville relations for polytopes, spheres and Eulerian partially ordered sets, Invent. Math. 79 (1985), 143-157.

[BE] L. Billera and R. Ehrenborg, Monotonicity properties of the cd-index for polytopes, Math. Z. 233 (2000), 421-441.

[BK] M. Bayer and A. Klapper, A new index for polytopes, Discrete Comput. Geom. 6 (1991), 33-47.

[BL] L. Billera and N. Liu, Noncommutative enumeration in graded posets, J. Algebraic Combin., to appear.

[BS] N. Bergeron and F. Sottile, Hopf algebras and edge-labeled posets, J. Algebra 216 (1999), 641-651.

[BMSV] N. Bergeron, S. Mykytiuk, F. Sottile and S. Van Willigenburg, Noncommutative Pieri operations on posets, J. Combin. Theory Ser. A 91 (2000), 84-110.

[E1] R. Ehrenborg, On Posets and Hopf algebras, Adv. Math. 119 (1996), 1-25.

[E2] R. Ehrenborg, On $k$-Eulerian posets, preprint (2000).

[EH] R. Ehrenborg and G. Hetyei, Newtonian colgebras, preprint (1998).

[ER] R. Ehrenborg and M. Readdy, Coproducts and the cd-index, J. Algebraic Combin. 8 (1998), 273-299.

[JR] S. A. Joni and G. C. Rota, Coalgebras and bialgebras in combinatorics, Stud. Appl. Math. 61 (1979), 93-139. Reprinted in Gian-Carlo Rota on Combinatorics: Introductory Papers and Commentaries (J. P. S. Kung, ed.), Birkhäuser, Boston (1995).

[S1] W. Schmitt, Incidence Hopf algebras, J. Pure Appl. Algebra 96 (1994), 299-330.

[S2] R. P. Stanley, Flag f-vectors and the cd-index, Math. Z. 216 (1994), 483-499.

[S3] R. P. Stanley, Flag-Symmetric and locally rank-symmetric partially ordered sets, Electron. J. Combin. 3(2) (1996), \#R6.

Received October 22, 2000, and in revised form January 4, 2001, and February 6, 2001.

Online publication November 12, 2001. 